\title{
The IRF2BP2-KLF2 axis regulates osteoclast and osteoblast differentiation
}

\author{
Inyoung Kim ${ }^{1}$, Jung Ha Kim ${ }^{1}$, Kabsun Kim ${ }^{1}$, Semun Seong ${ }^{1,2}$ E Nacksung Kim ${ }^{1,2, *}$ \\ Departments of ${ }^{1}$ Pharmacology, ${ }^{2}$ Biomedical Sciences, Chonnam National University Medical School, Gwangju 61469, Korea
}

\begin{abstract}
Kruppel-like factor 2 (KLF2) has been implicated in the regulation of cell proliferation, differentiation, and survival in a variety of cells. Recently, it has been reported that KLF2 regulates the p65-mediated transactivation of NF- $\mathrm{kB}$. Although the NF-KB pathway plays an important role in the differentiation of osteoclasts and osteoblasts, the role of KLF2 in these bone cells has not yet been fully elucidated. In this study, we demonstrated that KLF2 regulates osteoclast and osteoblast differentiation. The overexpression of KLF2 in osteoclast precursor cells inhibited osteoclast differentiation by downregulating c-Fos, NFATc1, and TRAP expression, while KLF2 overexpression in osteoblasts enhanced osteoblast differentiation and function by upregulating Runx2, ALP, and BSP expression. Conversely, the downregulation of KLF2 with KLF2-specific siRNA increased osteoclast differentiation and inhibited osteoblast differentiation. Moreover, the overexpression of interferon regulatory protein 2-binding protein 2 (IRF2BP2), a regulator of KLF2, suppressed osteoclast differentiation and enhanced osteoblast differentiation and function. These effects were reversed by downregulating KLF2. Collectively, our data provide new insights and evidence to suggest that the IRF2BP2/KLF2 axis mediates osteoclast and osteoblast differentiation, thereby affecting bone homeostasis. [BMB Reports 2019; 52(7): 469-474]
\end{abstract}

\section{INTRODUCTION}

Bone homeostasis is the process by which bone is continually renewed by bone-resorbing osteoclasts and bone-forming osteoblasts. The balance between osteoclasts and osteoblasts is crucial for maintaining bone and determining bone density. However, imbalances between these bone cells have been associated with various diseases, such as osteoporosis and rheumatoid arthritis. Therefore, it is important to identify the

*Corresponding author. Tel: +82-61-379-2835; Fax: +82-61-3736974; E-mail: nacksung@jnu.ac.kr

https://doi.org/10.5483/BMBRep.2019.52.7.104

Received 8 April 2019, Revised 23 April 2019, Accepted 25 May 2019

Keywords: IRF2BP2, KLF2, NF-кB, Osteoblast, Osteoclast molecules that regulate both osteoclast and osteoblast differentiation to maintain bone homeostasis.

Two essential cytokines, macrophage colony-stimulating factor (M-CSF) and receptor activator of nuclear factor-kappa B (NF-kB) ligand (RANKL), induce osteoclast differentiation. RANKL stimulation activates components of the NF- $\mathrm{KB}$ and MAPK signaling pathways, such as ERK, JNK, and p38 MAPKs (1). In addition, RANKL activates various transcription factors including c-Fos and nuclear factor of activated $\mathrm{T}$ cells (NFATc1) (2). c-Fos regulates the induction of NFATc1 expression by RANKL, and the cooperation between c-Fos and NFATc1 induces the expression of target genes, such as tartrate resistant acid phosphatase (TRAP) and osteoclast associated receptor (OSCAR) (3).

Osteoblasts are mononuclear cells that differentiate from mesenchymal cells due to the activation of various transcription factors (4). Bone morphogenetic protein 2 (BMP2) plays an important role in osteoblast differentiation (5). Runt-related transcription factor 2 (Runx2) induces the expression of various osteogenic genes, such as alkaline phosphatase (ALP), osteocalcin, and bone sialoprotein (BSP) (6).

$N F-\kappa B$ is a family of five transcription factors consisting of

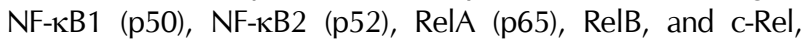
which are involved in the differentiation of osteoblasts and osteoclasts (7-9). Osteoclast differentiation is impaired in NF- $\kappa B$ double knockout precursor cells (10). In osteoblasts, it has been reported that the p65 subunit of NF- $\mathrm{KB}$ suppresses the DNA binding activity of Smad proteins during BMP2-induced osteoblast differentiation, resulting in the inhibition of bone formation by NF-kB (11). Furthermore, NF- $\kappa B$ suppression prevents osteoporotic bone loss while NF- $\kappa$ B RelB inhibits osteoblast differentiation and bone formation by regulating Runx2 $(7,12)$. Moreover, it is known that mammalian sterile 20-like kinase 2 (Mst2) modulates bone homeostasis by enhancing osteoblast differentiation and attenuating osteoclast differentiation via the NF-kB pathway (13).

Kruppel-like factors (KLFs) are a subclass of zinc-finger transcription factors that regulate cellular growth, differentiation, and inflammation (14). KLF2, originally known as lung KLF, plays an important regulatory role in aspects of hematopoietic cell biology such as cell quiescence, proliferation, differentiation, and survival (15). Furthermore, KLF2 negatively

ISSN: 1976-670X (electronic edition)

Copyright (C) 2019 by the The Korean Society for Biochemistry and Molecular Biology

c) This is an open-access article distributed under the terms of the Creative Commons Attribution Non-Commercial License (http://creativecommons.org/licenses/by-nc/4.0) which permits unrestricted non-commercial use, distribution, and reproduction in any medium, provided the original work is properly cited. 
regulates monocyte activation and function by inhibiting pro-inflammatory gene expression and reducing NF-KB promoter activity (16). Additionally, in KLF2 hemizygous mice, bone marrow-derived monocytes show an enhanced ability to differentiate into osteoclasts (17). These findings suggest that KLF2 may inhibit RANKL-induced osteoclast differentiation; however, the mechanisms involved are unknown and the role of KLF2 in osteoblasts has not been elucidated.

Interferon regulatory factor 2-binding protein 2 (IRF2BP2) consists of an $\mathrm{N}$-terminal $\mathrm{C} 4$ zinc finger and a C-terminal C3HC4 RING domain and acts as a co-repressor of IRF2 (18). IRF2BP2 acts as an activator of VEGFA expression by interacting with the Vgll4/TEAD4/MEF2 complex, and as an activator of the function of the repressor (18-20). In addition, IRF2BP2 regulates macrophage inflammation and lipid homeostasis by increasing MEF2-dependent KLF2 expression (21). IRF2BP2 is expected to control the differentiation of bone cells through KLF2, but its roles in bone remodeling have not yet been revealed.

In this study, we investigated the roles of KLF2, which suppresses the transcription factor NF-kB, in osteoclast differentiation and osteoblast differentiation and function. KLF2 was shown to inhibit osteoclast differentiation, whilst enhancing osteoblast differentiation and function. In addition, IRF2BP2 suppressed osteoclast differentiation and promoted osteoblast differentiation. Therefore, our data suggest that the IRF2BP2/KLF2 axis is important for osteoclastogenesis and osteoblastogenesis.

\section{RESULTS}

\section{KLF2 inhibits RANKL-induced osteoclast differentiation}

To investigate the expression of KLF2 during osteoclast differentiation, BMMs were cultured with M-CSF and RANKL for 3 days. The expression of osteoclast marker genes such as c-Fos, NFATc1, and TRAP was significantly increased during osteoclast differentiation (Supplementary Fig. 1A). KLF2 was expressed at a low level in BMMs on day 1, with KLF2 expression gradually increasing during RANKL-induced osteoclast differentiation (Supplementary Fig. 1A).

To investigate the role of KLF2 in osteoclastogenesis, KLF2 was overexpressed in BMMs by retroviral transduction. RANKL induced osteoclast differentiation in the control vector-infected BMMs, whereas the number of mature osteoclasts was significantly decreased in BMMs compared to the control cells, when KLF2 was overexpressed (Fig. 1A). Next, we examined whether KLF2 overexpression affected the expression of marker genes. Compared with the control cells, overexpression of inhibited the mRNA expression of c-Fos, NFATC1, and TRAP (Fig. 1B), and reduced the protein expression of c-Fos and NFATc1 (Fig. 1C).

Since KLF2 overexpression inhibited RANKL-induced osteoclastogenesis, we examined the physiological role of KLF2 during osteoclastogenesis using siRNA. Expression of
KLF2 was significantly decreased in BMMs transfected with KLF2-specific siRNA (Fig. 1D). KLF2 downregulation in BMMs enhanced osteoclast differentiation (Fig. 1E) and increased the mRNA expression of c-Fos, NFATc1, and TRAP (Fig. 1F) and the protein levels of c-Fos and NFATc1 (Fig. 1G). These results indicate that KLF2 acts as a negative regulator during RANKL-induced osteoclast differentiation.

\section{KLF2 positively regulates osteoblast differentiation and function}

Next, we investigated KLF2 expression during osteoblast differentiation. Primary calvarial osteoblasts were cultured in osteogenic medium (OGM) containing BMP2, $\beta$-glycerophosphate, and ascorbic acid. The expression of Rnux2, ALP, and BSP increased during osteoblast differentiation (Supplementary Fig. 1B). KLF2 was expressed in osteoblast precursor cells and

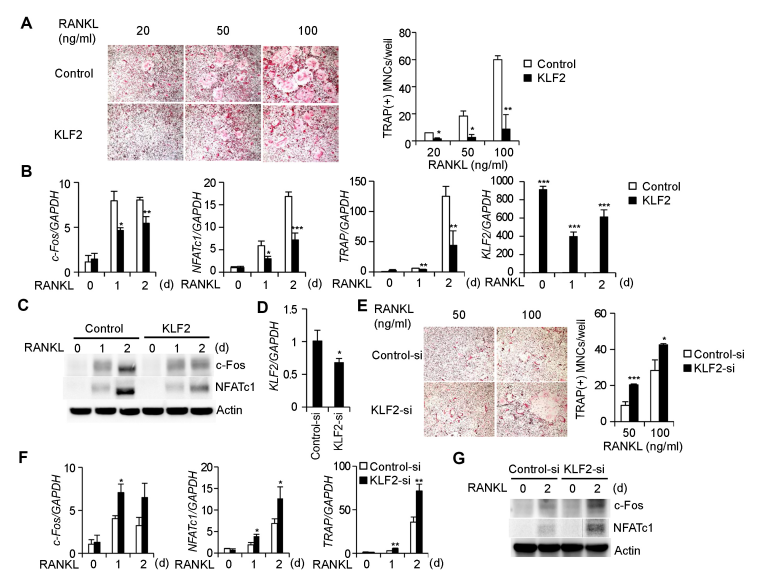

Fig. 1. KLF2 inhibits RANKL-induced osteoclast differentiation. (A) BMMs were transduced with pMX-IRES-EGFP (control) or KLF2 retroviruses and cultured in the presence of M-CSF and various RANKL concentrations for 3 days. Cultured cells were stained for TRAP (left panel). The number of TRAP-positive multinucleate cells (MNCs) per well was counted (right panel). (B, C) BMMs were transduced with either pMX-IRES-EGFP (control) or KLF2 retroviruses and cultured in the presence of M-CSF and RANKL for the indicated length of time. (B) The mRNA levels of c-Fos, NFATC1, TRAP, and KLF2 were assessed by real-time PCR. (C) Cell lysates were harvested from cultured cells and immunoblotted with the indicated antibodies. (D-G) BMMs were transfected with either control or KLF2 siRNAs. (D) The mRNA level of KLF2 was assessed by real-time PCR. (E) Transfected BMMs were cultured in the presence of M-CSF and various RANKL concentrations for 3 days. Cultured cells were then stained for TRAP (left panel). The number of TRAP-positive MNCs per well was counted (right panel). (F, G) Transfected BMMs were cultured in the presence of M-CSF and RANKL for the indicated length of time. (F) The mRNA levels of c-Fos, NFATc1, and TRAP were assessed by real-time PCR. (G) Cell lysates were harvested from cultured cells and immunoblotted with the indicated antibodies. Data represent the mean \pm standard deviation (SD) of triplicate samples. ${ }^{* P}<0.05, * * P<0.01$, and $* * * \mathrm{P}<0.001$ versus the control. 
KLF2 expression gradually increased during osteoblastogenesis (Supplementary Fig. 1B).

To investigate the role of KLF2 in osteoblasts, we overexpressed KLF2 in primary osteoblast precursors cells. The differentiation and function of osteoblasts were measured by ALP activity and bone nodule formation. ALP activity was significantly increased in KLF2-overexpressing osteoblasts compared with the control (Fig. 2A), whilst KLF2 overexpression significantly increased the nodule formation and alizarin red activity (Fig. 2B). Next, we examined whether KLF2 affected the expression of osteoblast marker genes. Overexpression of KLF2 significantly increased the expression of Runx2, ALP, and BSP during osteoblast differentiation compared to the control (Fig. 2C).

The physiological role of KLF2 in osteoblasts was investigated using KLF2-specific siRNA. When primary osteoblast precursor cells were transfected with KLF2 siRNA, KLF2 expression was significantly decreased (Fig. 2D). The

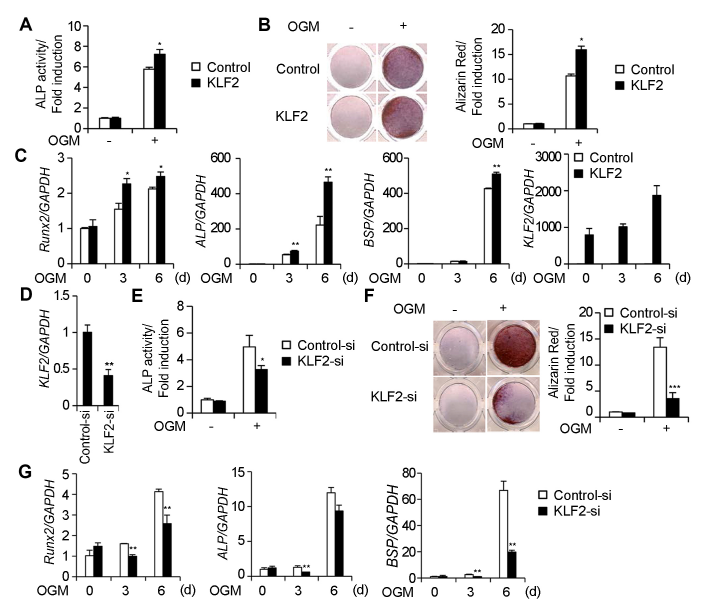

Fig. 2. KLF2 enhances osteoblast differentiation and function. (A-C) Osteoblasts were transduced with either pMX-IRES-EGFP (control) or KLF2 retroviruses and cultured in an osteogenic medium. (A) Cells cultured for 3 days were subjected to an alkaline phosphatase (ALP) activity assay. ALP activity was measured as the change in the absorbance at $405 \mathrm{~nm}$. (B) Cells cultured for 9 days were fixed and stained for alizarin red (left panel), which was quantified by densitometry at $562 \mathrm{~nm}$ (right panel). (C) The mRNA levels of Runx2, ALP, BSP, and KLF2 were assessed by real-time PCR. (D-G) Osteoblasts were transfected with either control or KLF2 siRNAs and cultured in an osteogenic medium. (D) The mRNA level of KLF2 was assessed by real-time PCR. (E) Cells cultured for 3 days were subjected to an ALP activity assay. ALP activity was measured as the change in the absorbance at $405 \mathrm{~nm}$. (F) Cells cultured for 9 days were fixed and stained for alizarin red (left panel), which was quantified by densitometry at $562 \mathrm{~nm}$ (right panel). (G) Transfected cells were cultured for the indicated length of time and the mRNA expression of Runx2, ALP, and BSP was assessed by real-time PCR analysis. Data represent the mean \pm standard deviation (SD) of triplicate samples. $* P<0.05, * * P<0.01$, and $* * * P<$ 0.001 versus the control. inhibition of KLF2 expression by KLF2-specific siRNA significantly reduced ALP activity (Fig. 2E), bone nodule formation, and alizarin red activity (Fig. 2F) compared to the control. During osteoblast differentiation, the expression of osteoblast marker genes was significantly decreased when KLF2 expression was reduced by siRNA (Fig. 2G). These data demonstrate that KLF2 positively regulates osteoblast differentiation and function.

\section{IRF2BP2, a KLF2 regulator, mediates osteoclast and osteoblast differentiation}

It has been reported that IRF2BP2 is a novel regulator of KLF2 (21); therefore, we examined whether IRF2BP2 could regulate

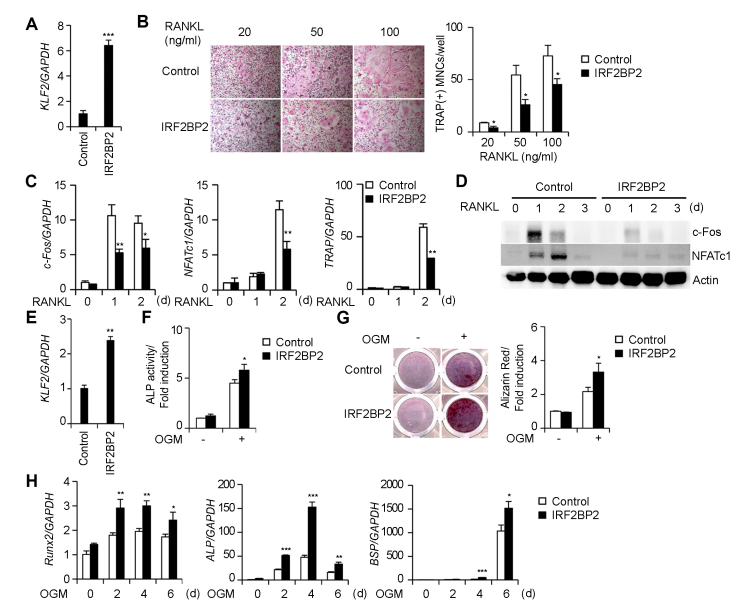

Fig. 3. Interferon regulatory factor 2-binding protein 2 (IRF2BP2) regulates the differentiation of osteoclasts and osteoblasts. (A-D) Bone marrow-derived macrophage cells (BMMs) were transduced with either pMX-IRES-EGFP (control) or IRF2BP2 retroviruses. (A) The mRNA level of KLF2 was assessed by real-time PCR. (B) Transduced BMMs were cultured in the presence of M-CSF and various RANKL concentrations for 3 days. Cultured cells were stained for TRAP (left panel). The number of TRAP-positive multinucleate cells (MNCs) per well was counted (right panel). (C, D) Transduced BMMs were cultured in the presence of M-CSF and RANKL for the indicated length of time. (C) Transduced cells were cultured for the indicated length of time and the mRNA expression of c-Fos, NFATC1, and TRAP was assessed by real-time PCR. (D) Cell lysates were harvested from cultured cells and immunoblotted with the indicated antibodies. (E-H) Osteoblasts were transduced with either pMX-IRES-EGFP (control) or IRF2BP2 retroviruses. (E) The mRNA level of KLF2 was assessed by real-time PCR. (F-H) Transduced osteoblasts were cultured in an osteogenic medium. (F) Cells cultured for 3 days were fixed and subjected to an alkaline phosphatase (ALP) activity assay. ALP activity was measured by densitometry at $405 \mathrm{~nm}$. (G) Cells cultured for 9 days were fixed and stained for alizarin red (left panel). Alizarin red staining activity was quantified by densitometry at $562 \mathrm{~nm}$ (right panel). $(\mathrm{H})$ Transduced cells were cultured for the indicated length of time and the mRNA expression of Runx2, ALP, and BSP was assessed by real-time PCR. Data represent the mean \pm standard deviation (SD) of triplicate samples. ${ }^{*} \mathrm{P}<0.05,{ }^{*} \mathrm{P}<0.01$, and $* * * P<$ 0.001 versus the control. 
KLF2 expression in osteoclasts. IRF2BP2 overexpression significantly increased KLF2 expression in BMMs (Fig. 3A), suggesting that IRF2BP2 acts upstream of KLF2 in osteoclasts. However, as the role of IRF2BP2 is unknown in osteoclasts, we examined whether IRF2BP2 affected osteoclast differentiation. IRF2BP2 overexpression significantly inhibited osteoclast differentiation in BMMs compared to the control (Fig. 3B). During osteoclast differentiation, IRF2BP2 overexpression inhibited the mRNA expression of c-Fos, NFATc1, and TRAP (Fig. 3C), and attenuated the protein expression of C-Fos and NFATc1 (Fig. 3D). These data indicate that IRF2BP2 inhibits osteoclast differentiation in a manner similar to KLF2.

Since the role of IRF2BP2 in osteoblasts has not yet been reported, we investigated the effect of IRF2BP2 on osteoblast differentiation and function. Similar to its effect in osteoclasts, IRF2BP2 overexpression led to increased KLF2 expression in preosteoblasts (Fig. 3E). Next, we examined the effect of IRF2BP2 on osteoblast differentiation and function. IRF2BP2 overexpression in osteoblasts significantly enhanced ALP activity (Fig. 3F), nodule formation, and alizarin red activity (Fig. 3G) compared to the control. Furthermore, IRF2BP2 overexpression significantly increased the expression of Runx2, ALP, and BSP compared to the control (Fig. 3H). Thus, these data demonstrate that IRF2BP2 enhances osteoblast differentiation and function in a similar manner to KLF2. Collectively, our data suggest that IRF2BP2 may act upstream of KLF2 in bone cells to regulate the differentiation of these cells.

\section{IRF2BP2 regulates osteoclast and osteoblast differentiation via KLF2}

We hypothesized that IRF2BP2 might regulate the differentiation of osteoclasts and osteoblasts via KLF2. We first examined whether the reduction in osteoclast differentiation caused by IRF2BP2 overexpression could be restored by downregulating KLF2. The overexpression of IRF2BP2 in BMMs significantly reduced osteoclast differentiation (Fig. 4A), and the inhibitory effect of IRF2BP2 was significantly reduced by downregulating KLF2 using siRNA (Fig. 4A). The reduction in c-Fos expression by IRF2BP2 overexpression was reversed by downregulating KLF2 using siRNA (Fig. 4B). These results indicate that IRF2BP2 regulates osteoclast differentiation via KLF2.

Next, we examined whether IRF2BP2 enhanced osteoblast differentiation and function via KLF2. IRF2BP2 overexpression significantly increased ALP activity (Fig. 4C), nodule formation, and alizarin red activity (Fig. 4D); however, these effects were significantly reduced by downregulating KLF2 using siRNA (Fig. 4C, D). The increase in Runx2 expression caused by IRF2BP2 overexpression was reduced by downregulating KLF2 using siRNA (Fig. 4E). These data indicate that IRF2BP2 mediates osteoblast differentiation and function via KLF2. Taken together, these results suggest that IRF2BP2 controls osteoclast and osteoblast differentiation via KLF2.

\section{DISCUSSION}

The bone marrow-derived monocytes of KLF2 hemizygous mice display elevated function and differentiation into mature osteoclasts compared to those of wild-type mice (17). KLF2 is recruited to $\mathrm{P} 300 / \mathrm{CBP}$-associated factor (PCAF) and inhibits the transcriptional activity of $\mathrm{NF}-\kappa \mathrm{B}$, thereby reducing the expression of several inflammatory genes and cytokines (22, 23). It is known that NF-kB promotes osteoclast differentiation and inhibits osteoblast differentiation $(24,25)$. Previous reports have suggested that KLF2 negatively regulates osteoclast differentiation; however, the mechanisms by which KLF2 acts have not yet been elucidated and the role of KLF2 in osteoblasts remains unknown.

In this study, we report that KLF2 plays different roles in the differentiation of osteoclasts and osteoblasts. We showed that KLF2 overexpression in BMMs suppresses RANKL-induced osteoclast differentiation, whereas the downregulation of KLF2 using siRNA increased osteoclast formation. In contrast, KLF2 overexpression in osteoblast precursor cells increased osteoblast differentiation and function, while KLF2 downregulation decreased the formation and function of osteoblasts. These data suggest that KLF2 negatively regulates osteoclast differentiation and positively regulates osteoblast differentiation. Although KLF2 does not affect p65 or IKB kinase (IKK) expression and nuclear accumulation, or the phosphorylation

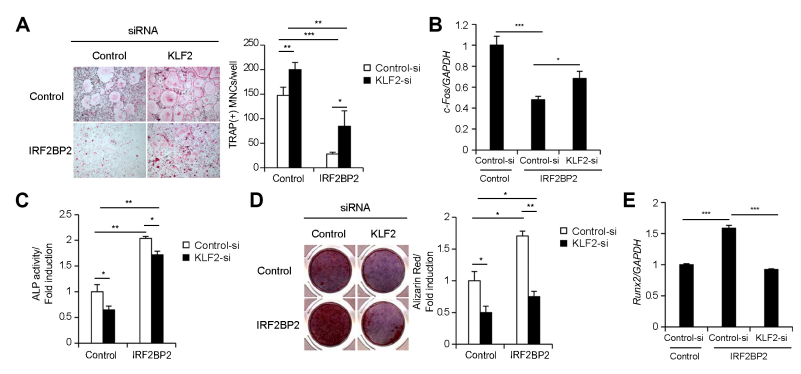

Fig. 4. IRF2BP2 regulates osteoclastogenesis and osteoblastogenesis via KLF2. (A, B) Bone marrow-derived macrophage cells (BMMs) were transfected with control or KLF2 siRNAs. Transfected BMMs were transduced with pMX-IRES-EGFP (control) or IRF2BP2 retroviruses and cultured with M-CSF and RANKL. (A) Cultured cells were fixed and stained for TRAP (left panel). The number of TRAP-positive multinucleate cells (MNCs) per well was counted (right panel). (B) C-Fos mRNA expression was assessed by real-time PCR. (C-E) Osteoblasts were transfected with control or KLF2 siRNAs, then transduced with pMX-IRES-EGFP (control) or IRF2BP2 retroviruses and cultured in an osteogenic medium. Cells cultured for 3 days were fixed and subjected to an alkaline phosphatase (ALP) activity assay. ALP activity was measured by densitometry at $405 \mathrm{~nm}$. (D) Cells cultured for 9 days were fixed and stained for alizarin red (left panel). Alizarin red staining activity was quantified by densitometry at $562 \mathrm{~nm}$ (right panel). (E) Runx2 mRNA expression was assessed by real-time PCR. Data represent the mean \pm standard deviation (SD) of triplicate samples. $* P<0.05, * * P<0.01$, and $* * * P<0.001$ versus the control. 
and degradation of $1 \kappa \mathrm{B}$, it has been reported that it interacts directly with PCAF, the coactivator of NF-kB, to suppress p65 transcriptional activity $(16,23)$. In fact, we observed that the overexpression of KLF2 in osteoclasts did not change the phosphorylation and degradation of $\mathrm{I} \mathrm{K} B$ and that p65mediated transcriptional activity was inhibited by KLF2 in 293T cells (data not shown). Therefore, these results suggest that KLF2 may mediate the differentiation of osteoclasts and osteoblasts by suppressing the activity of the NF- $\mathrm{kB}$ promoter.

KLF2 plays an important role in regulating the activity of various immune cells and inhibiting the activation of monocytes (26). KLF2 is highly expressed in monocytes, and its expression is reduced by LPS stimulation in THP-1 monocyte-like cells and is also decreased when these cells differentiate into macrophages (16). KLF2 inhibits proinflammatory gene expression and suppresses the function and phagocytic ability of monocytes $(16,17)$. KLF2 mRNA levels were the most abundant during the BMM stage; it decreased sharply following RANKL treatment. KLF2 seems to have a negative regulatory role in osteoclastogenesis, which was clearly evidenced by overexpression or knockdown experiments. Therefore, the high level of KLF2 in BMMs may have a role in maintaining these cells in the un-differentiated stages. Further studies are needed to elucidate the role of KLF2 in BMMs.

Chen et al. reported that KLF2 expression was found to be significantly reduced in IRF2BP2-deficient bone marrowderived macrophages $(21,27)$. Using ChIP and reporter assays, they showed that IRF2BP2 binds to the promoter of KLF2, resulting in the upregulation of KLF2 expression (21). We found that the overexpression of IRF2BP2 in BMMs and preosteoblasts increased KLF2 expression, suggesting that IRF2BP2 acts upstream of KLF2 in bone cells. We also showed that IRF2BP2 overexpression inhibits osteoclast differentiation and promotes osteoblast differentiation and function in the same manner as KLF2. In addition, we observed that the IRF2BP2-mediated inhibition of osteoclast differentiation and c-Fos expression was restored by downregulating KLF2, and that the IRF2BP2-mediated enhancement of osteoblast differentiation and Runx2 expression was rescued by downregulating KLF2. Taken together, our results suggest that IRF2BP2 acts upstream of KLF2 in osteoclasts and osteoblasts, and that the IRF2BP2/KLF2 axis regulates bone homeostasis by regulating the transcriptional activity of NF- $\mathrm{\kappa B}$.

IRF2BP2 interacts with NFATc2 and inhibits the NFATc2mediated transactivation of the IL-2 and IL-4 promoters and the TNF- $\alpha \kappa 3$ element (19). NFATc2 is an upstream regulator of NFATc1, which affects osteoclast differentiation by binding the NFATc1 promoter $(28,29)$. Therefore, IRF2BP2 may directly modulate NFATC1 in a KLF2-independent manner to control osteoclast differentiation.

In conclusion, we demonstrated that KLF2 inhibits osteoclast differentiation by reducing c-Fos expression, whilst KLF2 promotes the differentiation and function of osteoblasts by increasing Runx2 expression. We also found that IRF2BP2 regulates KLF2 in bone cells. Therefore, our results suggest that the IRF2BP2/KLF2 signaling pathway plays an important role in the regulation of bone cells via the transcriptional activity of $\mathrm{NF}-\kappa \mathrm{B}$, and that the IRF2BP2/KLF2/NF- $\mathrm{KB}$ axis could be a potential therapeutic target for various bone diseases.

\section{MATERIALS AND METHODS}

See supplementary information for Material and Methods. Kindly refer to the supplementary information section for the Material and Methods used in this study.

\section{ACKNOWLEDGEMENTS}

This work was supported by the National Research Foundation of Korea (NRF) grants funded by the Korea government (MIST) (No. 2019R1A5A2027521 and 2017R1A6A3A11031114).

\section{CONFLICTS OF INTEREST}

The authors have no conflicting interests.

\section{REFERENCES}

1. Leibbrandt $A$ and Penninger JM (2008) RANK/RANKL: regulators of immune responses and bone physiology. Ann N Y Acad Sci 1143, 123-150

2. Kim JH and Kim N (2016) Signaling Pathways in Osteoclast Differentiation. Chonnam Med J 52, 12-17

3. Takayanagi H, Kim S, Koga T et al (2002) Induction and activation of the transcription factor NFATc1 (NFAT2) integrate RANKL signaling in terminal differentiation of osteoclasts. Dev Cell 3, 889-901

4. Katagiri T and Takahashi N (2002) Regulatory mechanisms of osteoblast and osteoclast differentiation. Oral Dis 8, $147-159$

5. Matsubara T, Kida K, Yamaguchi A et al (2008) BMP2 regulates Osterix through Msx2 and Runx2 during osteoblast differentiation. J Biol Chem 283, 29119-29125

6. Schroeder TM, Jensen ED and Westendorf JJ (2005) Runx2: a master organizer of gene transcription in developing and maturing osteoblasts. Birth Defects Res C Embryo Today 75, 213-225

7. Boyce BF, Yao Z and Xing L (2010) Functions of nuclear factor kappaB in bone. Ann N Y Acad Sci 1192, 367-375

8. Kim I, Kim JH, Kim K, Seong S and Kim N (2017) Tusc2/Fus1 regulates osteoclast differentiation through NF-kappaB and NFATc1. BMB Rep 50, 454-459

9. Bak SU, Kim S, Hwang HJ et al (2017) Heme oxygenase-1 (HO-1)/carbon monoxide (CO) axis suppresses RANKL-induced osteoclastic differentiation by inhibiting redox-sensitive NF-kappaB activation. BMB Rep 50, 103-108

10. Yamashita T, Yao Z, Li F et al (2007) NF-kappaB p50 and p52 regulate receptor activator of NF-kappaB ligand (RANKL) and tumor necrosis factor-induced osteoclast 
precursor differentiation by activating c-Fos and NFATc1. J Biol Chem 282, 18245-18253

11. Yamazaki M, Fukushima $H$, Shin $M$ et al (2009) Tumor necrosis factor alpha represses bone morphogenetic protein (BMP) signaling by interfering with the DNA binding of Smads through the activation of NF-kappaB. J Biol Chem 284, 35987-35995

12. Yao Z, Li Y, Yin X, Dong $Y$, Xing $L$ and Boyce BF (2014) NF-kappaB RelB negatively regulates osteoblast differentiation and bone formation. J Bone Miner Res 29, 866-877

13. Lee J, Youn BU, Kim K et al (2015) Mst2 Controls Bone Homeostasis by Regulating Osteoclast and Osteoblast Differentiation. J Bone Miner Res 30, 1597-1607

14. Nayak L, Goduni L, Takami Y et al (2013) Kruppel-like factor 2 is a transcriptional regulator of chronic and acute inflammation. Am J Pathol 182, 1696-1704

15. Dang DT, Pevsner J and Yang VW (2000) The biology of the mammalian Kruppel-like family of transcription factors. Int J Biochem Cell Biol 32, 1103-1121

16. Das H, Kumar A, Lin Z et al (2006) Kruppel-like factor 2 (KLF2) regulates proinflammatory activation of monocytes. Proc Natl Acad Sci U S A 103, 6653-6658

17. Das M, Lu J, Joseph M et al (2012) Kruppel-like factor 2 (KLF2) regulates monocyte differentiation and functions in mBSA and IL-1beta-induced arthritis. Curr Mol Med 12, 113-125

18. Childs KS and Goodbourn S (2003) Identification of novel co-repressor molecules for Interferon Regulatory Factor-2. Nucleic Acids Res 31, 3016-3026

19. Carneiro FR, Ramalho-Oliveira R, Mognol GP and Viola JP (2011) Interferon regulatory factor 2 binding protein 2 is a new NFAT1 partner and represses its transcriptional activity. Mol Cell Biol 31, 2889-2901

20. Teng AC, Kuraitis D, Deeke SA et al (2010) IRF2BP2 is a skeletal and cardiac muscle-enriched ischemia-inducible activator of VEGFA expression. FASEB J 24, 4825-4834

21. Chen HH, Keyhanian K, Zhou X et al (2015) IRF2BP2 Reduces Macrophage Inflammation and Susceptibility to Atherosclerosis. Circ Res 117, 671-683

22. Jha $P$ and Das $H$ (2017) KLF2 in Regulation of NF-kappaB-Mediated Immune Cell Function and Inflammation. Int J Mol Sci 18, 2383-2420

23. SenBanerjee S, Lin Z, Atkins GB et al (2004) KLF2 Is a novel transcriptional regulator of endothelial proinflammatory activation. J Exp Med 199, 1305-1315

24. Boyce BF, Xiu Y, Li J, Xing L and Yao Z (2015) NF-kappaB-Mediated Regulation of Osteoclastogenesis. Endocrinol Metab (Seoul) 30, 35-44

25. Chang J, Wang Z, Tang E et al (2009) Inhibition of osteoblastic bone formation by nuclear factor-kappaB. Nat Med 15, 682-689

26. Cao Z, Sun X, Icli B, Wara AK and Feinberg MW (2010) Role of Kruppel-like factors in leukocyte development, function, and disease. Blood 116, 4404-4414

27. Zhang H and Reilly MP (2015) IRF2BP2: A New Player at the Crossroads of Inflammation and Lipid Metabolism. Circ Res 117, 656-658

28. Zhou B, Cron RQ, Wu B et al (2002) Regulation of the murine Nfatc1 gene by NFATc2. J Biol Chem 277, 10704-10711

29. Ikeda F, Nishimura R, Matsubara $T$ et al (2004) Critical roles of C-Jun signaling in regulation of NFAT family and RANKL-regulated osteoclast differentiation. J Clin Invest $114,475-484$ 